\title{
Gekonnt reagieren, wenn der Patient wütend ist
}

\author{
Verärgerte Patienten kosten Praxisteams Zeit und Kraft, schlimmsten- \\ falls auch Umsatz. Mit der richtigen Strategie kann Ärger aber souverän \\ und leicht aus der Welt geschafft werden.
}

\footnotetext{
$\mathrm{P}$
} atientenbeschwerden kommen hin und wieder in jeder Arztpraxis vor. Wer unvorbereitet in eine Beschwerdesituation gerät, steht dem verärgerten Patienten oft unsicher oder hilflos gegenüber. Mit der richtigen $\mathrm{Ge}$ sprächsführung gelingt es aber in vielen Fällen, derartige Situationen professionell zu meistern. Wichtigster Punkt hierbei: Werten Sie die Beschwerde nicht automatisch als Angriff auf die eigene Person. Sehen Sie es vielmehr als Chance, dass der Patient seine Unzufriedenheit offen ausspricht und nicht einfach kommentarlos zu einer anderen Arztpraxis wechselt. Gleichzeitig ist jede Beschwerde auch eine wertvolle Rückmeldung zur Verbesserung der Qualität und des Service in der Arztpraxis.

\section{Zuhören und ausreden lassen}

Damit dies funktioniert, muss der Patient zunächst die Chance haben, seine Beschwerde vollständig zu schildern. Lassen Sie den Patienten daher ausreden ohne ihn zu unterbrechen oder ihm zu widersprechen. Wütende Patienten müssen zunächst „Dampf ablassen“, bevor eine sachliche Lösung gefunden werden kann. Zeigen Sie Verständnis für die Verärgerung des Patienten und drücken Sie dies auch durch ein gelegentliches Nicken oder die Bemerkung „Ich verstehe" aus. So fühlt sich der Patient ernst genommen. Erst wenn der Patient ausgeredet hat, sollten selber Fragen gestellt und bestenfalls auch eine Lösung angeboten werden. Bei berechtigten Beschwerden sollten Sie sich beim Patienten für den Fehler entschuldigen.
Es kommt vor, dass Patienten ihren Ärger sehr lautstark vortragen, und andere Patienten dadurch auf die Beschwerde aufmerksam werden. Die Bitte, etwas leiser zu sprechen, hat bei diesem emotionalen Patiententyp meist keinen Erfolg. In diesen Fällen empfiehlt es sich, den Patienten in einen getrennten Raum zu bitten. Medizinische Fachangestellte sollten einen Kollegen oder den Praxischef hinzuziehen, denn durch eine weitere Person versachlicht sich die Diskussion häufig sehr schnell.

\section{Beschwerdeformular}

Beschwerden sollten stets mit einer für den Patienten zufriedenstellenden Lösung enden. Sie können entweder selbst einen Vorschlag anbieten oder den Patienten fragen, wie aus seiner Sicht eine akzeptable Lösung aussehen würde. Der Patient sollte dabei nicht das Gefühl haben, dass ihm damit "großzügig“ ein Gefallen getan wird. Aus seiner Sicht ist die Bearbeitung der Beschwerde ja keine Kulanzlösung, sondern sein gutes Recht. Deshalb sollte man auch auf gönnerhafte Äußerungen wie „Das machen wir aber nur ausnahmsweise!" verzichten.

Jede Beschwerde sollte nach der Bearbeitung in der Praxis dokumentiert werden, auch wenn es einige Minuten Zeit kostet. Dazu kann entweder ein Beschwerdeformular oder ein Beschwerdebuch eingesetzt werden, in dem die Beschwerde mit geringerem Zeitaufwand stichwortartig festgehalten wird. In der nächsten Mitarbeiterbesprechung können die Reklamationen im Sinne der Qualitätsverbesserung diskutiert wer-

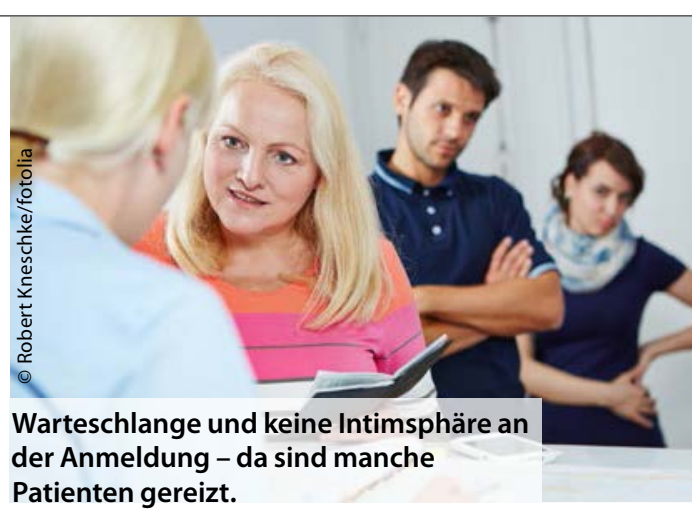

den. Dabei sollte besprochen werden, was im Einzelfall gut oder schlecht gelaufen ist, wie die bestmögliche Lösung ausgesehen hätte und wie derartige Situationen in Zukunft vermieden werden können. So verbessert sich das Beschwerdemanagement der Praxis kontinuierlich.

Unzufriedene Patienten machen ihren Ärger oft im Freundes- und Bekanntenkreis oder in Internetforen publik - und auf diese Weise negative Mund-zuMund-Propaganda. Wenn eine Beschwerde hingegen kulant behandelt wird, so berichtet der Patient auch darüber und der ursprünglich unschöne Anlass führt vielleicht sogar zu einer positiven Weiterempfehlung, weil die Arztpraxis als besonders patientenfreundlich dargestellt wird.

\section{Praxisinterner}

\section{Beschwerdestandard}

Jedes Praxisteam sollte gemeinsam eine einheitliche Vorgehensweise festlegen, wie generell mit unzufriedenen Patienten umzugehen ist, damit es nicht dem Zufall überlassen wird, wie einzelne Mitarbeiter auf Beschwerden reagieren. Ein einheitlicher Beschwerdestandard vermeidet vor allem instinktive oder emotionale Reaktionen und hilft, Beschwerden sachlich zu behandeln. Solche Verhaltensregeln könnten wie folgt aussehen:

-Patienten ausreden lassen,

- für den Hinweis bedanken,

- Verständnis und Bedauern ausdrücken (ggf. entschuldigen),

_Lösung anbieten oder Lösungswünsche des Patienten erfragen,

- Beschwerde bearbeiten,

- Beschwerde und Lösung dokumentieren (Formular oder Beschwerdebuch), -Beschwerde beim nächsten Teammeeting besprechen.

Birgit Hallman 